\title{
Importance of dye host on absorption, propagation losses, and amplified spontaneous emission for dye-doped polymer thin films
}

\author{
Hélène Goudket, Tran Hong Nhung, Buntha Ea-Kim, Gisèle Roger, and Michael Canva
}

\begin{abstract}
The absorption spectra of dye-doped polymer thin films made from a variety of five dyes and six matrices, either organic or organomineral, are analyzed to evaluate the residual absorption in the red wavelength tail and in particular at amplified spontaneous emission (ASE) wavelengths. An absorption cutoff wavelength is defined as the extrapolated wavelength at which the absorption losses are expected to become negligible compared to the structure losses. Such absorption-spectrum-extrapolated wavelengths are compared to the ASE wavelengths and found to correlate for most of the dye-matrix couples. The propagation losses of PM597-doped organic polymers are also measured and accordingly found to increase with the glass transition temperature of the host matrix. (c) 2006 Optical Society of America

OCIS codes: $160.3380,160.4890,160.4760,310.2790,310.6860$.
\end{abstract}

\section{Introduction}

Dye-doped polymer thin films have been extensively studied for the past six years as promising gain media. Indeed, they present the advantages of the large spectral range and tuning ability of the dyes ${ }^{1}$ and are easily structured into various devices by using different techniques. ${ }^{2-5}$ The materials used for the fabrication of these integrated laser devices fall into three categories: luminescent conjugated polymers, $, 7,7$ dyedoped organic, ${ }^{8,9}$ or organomineral solgel10,11 polymers. In this work we concentrate on the thin films of either dye-doped organic or organomineral polymers.

To optimize the performances of the integrated laser devices, it is necessary to choose properly the dye-matrix couples used. As is well known, the residual absorption in the fluorescence band redshifts the laser peak emitted by the sample, as seen in Fig. 1. Yet the residual absorption on the red side of the fluorescence band is often neglected as it is usually lower than the spectrometer resolution. Indeed, the

The authors are with the Laboratoire Charles Fabry de l'Institut d'Optique, CNRS UMR 8501, Bâtiment 503, Université d'Orsay Paris-Sud 11, 91403 Orsay Cedex, France. H. Goudket's e-mail address is hgoudket@free.fr. T. H. Nhung's permanent address is Institute of Physics and Electronics, Vietnamese Academy of Science and Technology, Hanoi, Vietnam.

Received 25 January 2006; revised 2 June 2006; accepted 3 June 2006; posted 5 June 2006 (Doc. ID 67408).

$0003-6935 / 06 / 297736-06 \$ 15.00 / 0$

(C) 2006 Optical Society of America absorption spectrum is measured through the thickness of the sample, which is approximately $1 \mu \mathrm{m}$, but the amplified spontaneous emission (ASE) of the sample will propagate in the thin film for a distance of the order of $1 \mathrm{~mm}$ or $1 \mathrm{~cm}$, which is 3 or 4 orders of magnitude larger than the thickness of the sample. Therefore the apparently negligible absorption values in the red tail of the spectrum, which are close to or inferior to the spectrometer's resolution, have, in fact, a strong influence over a $1 \mathrm{~cm}$ propagation distance and are often mistakenly neglected. It is important to estimate the propagation losses due to absorption as the wavelength of interest is shifted from the strong absorption band toward the actual transparency region and especially at a potential ASE wavelength, as the absorption spectrum plays a key role in defining the gain-loss ratio that determines the ASE emission wavelength.

To evaluate propagation losses in a dye-doped polymer fiber, Kruhlak and Kuzyk ${ }^{12,13}$ have measured by means of another novel method, side-illumination spectroscopy (SIF), the linear absorption of the red tail of the absorption band by measuring the transmitted fluorescence as a function of the distance from the point of illumination. This method has also been applied to thin films by Geetha et al. ${ }^{14}$ In this work we have developed a quick method to extrapolate the residual absorption of the red-tail absorption spectrum. Therefore we estimated the ASE wavelength with standard laboratory apparatus, requiring the 


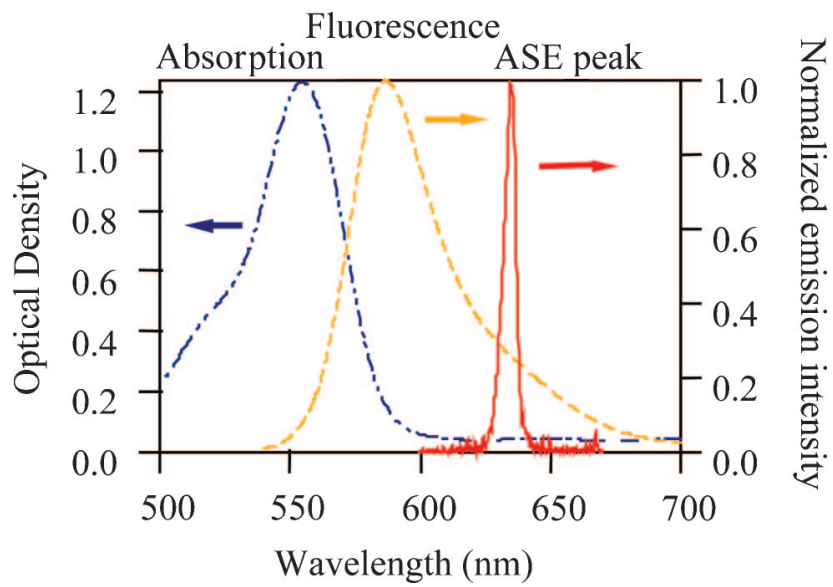

Fig. 1. (Color online) Absorption, fluorescence, and ASE emission spectra, which peak, respectively, at 555, 586, and $635 \mathrm{~nm}$, of a 2.3- $\mu$ m-thick RhB/TCPTEOS thin film.

use of only a spectrometer. We have studied the influence of the dye residual absorption present in the dye fluorescence spectral band on ASE emission and related it through a quantification of propagation losses at that ASE wavelength.

\section{Materials}

The materials studied in this work are a variety of dye-doped polymer samples. The dyes used were Rhodamine B (RhB), Rhodamine 6G (Rh6G), Pyrromethene 597 (PM597), 4-dicyanomethylene-2-methyl-6(p-dimethylaminostyryl)-4H-pyran (DCM), and Perylene Red (Pery Red).

The host matrices were either organic or organomineral solgel polymers. The organic polymers were poly (methyl methacrylate) (PMMA) of different average molecular weights, 15,000 g/mol (PMMA15), 120,000 $\mathrm{g} / \mathrm{mol}$ (PMMA120), and 350,000 g/mol (PMMA350), and poly(9-vinylcarbazole) (PVK) of an average molecular weight of $1,100,000 \mathrm{~g} / \mathrm{mol}$. These organic polymers can also be characterized by their glass transition temperatures $T_{g}$, respectively, $100{ }^{\circ} \mathrm{C}, 114{ }^{\circ} \mathrm{C}$, $122^{\circ} \mathrm{C}$, and $200{ }^{\circ} \mathrm{C}$, which are reported in Table 1 . The $T_{g}$ is the temperature at which the amorphous domains of a polymer take on the characteristic properties of the glass state: brittleness, stiffness, and rigidity. Thus the rigidity at ambient temperature of a polymer matrix is higher if it has a higher glass transition temperature. The organic polymers used were

Table 1. Average Molecular Weight and Glass Transition Temperature $T_{g}$ of the Organic Polymer Matrices

\begin{tabular}{ccc}
\hline & $\begin{array}{c}\text { Average Molecular } \\
\text { Weight } \\
(\mathrm{g} / \mathrm{mol})\end{array}$ & $\begin{array}{c}T_{g} \\
\left({ }^{\circ} \mathrm{C}\right)\end{array}$ \\
\hline Polymer Name & 15,000 & 100 \\
PMMA15 & 120,000 & 114 \\
PMMA120 & 350,000 & 122 \\
PMMA350 & $1,100,000$ & 200 \\
\hline
\end{tabular}

commercial powders (Aldrich) dissolved in dichloro1,2-ethane with the dye powders.

The other type of host matrix studied consisted of organomineral polymer matrices synthetized with the solgel process. These were thiocyanatopropyl(triethoxysilane) (TCPTEOS) and phenyl(triethoxysilane) (PhiTEOS). The dye powders were dissolved in the resulting sols.

The dye-doped polymer solutions were then spin coated on $25 \mathrm{~mm} \times 25 \mathrm{~mm}$ substrates at typical rotation speeds of 1000 to $4000 \mathrm{rpm}$ and heat treated for $10 \mathrm{~min}$ at $90{ }^{\circ} \mathrm{C}$ for the organic polymers and $30 \mathrm{~min}$ at $100^{\circ} \mathrm{C}$ for the solgel matrices, to form waveguiding thin films approximately $2 \mu \mathrm{m}$ thick. Fused-silica substrates (refractive index $n=1.46$ ) were used for the PMMA samples $(n \approx 1.49)$, while glass substrates $(n=1.51)$ were used for the solgel samples $(n \approx 1.54)$ for waveguiding considerations. The dye-matrix couples and the dye concentration and thickness of the samples are reported in Table 2, in columns 1 through 4 . Several series of each combination were prepared, and their absorption spectra were shown to be reproducible. It must be noted that RhB-doped and Rh6G-doped PVK samples were prepared but that they failed to show any ASE emission, probably due to dye-matrix interactions.

\section{Characterization}

\section{A. Absorption Spectrum}

The absorption spectra of the dye-doped polymer thin films were measured with a PerkinElmer Lambda 9-UV/VIS/NIR spectrophotometer. An analysis of the spectra was then made. Previous extensive studies on electro-optic dye-doped polymers in the 400 to $800 \mathrm{~nm}$ wavelength range have shown that the absorption coefficient's behavior in the high-wavelength tail of one absorption band can be modeled with a linear function in semilogarithmic representation. ${ }^{15}$ This enables us to extrapolate the absorption coefficient value up to hundreds of nanometers beyond what can be classically measured with thin-film transmission measurements. The slope of the linear fit is defined as

$$
\text { slope }=d\{\ln [\operatorname{Abs}(\lambda)]\} / d \lambda,
$$

where $\operatorname{Abs}(\lambda)$ is the absorption coefficient of the sample in $\mathrm{cm}^{-1}$, and the slope is expressed in $\mathrm{nm}^{-1}$. The absorption losses of propagating light are negligible if they are inferior to losses due to the structure of the waveguide, which we estimated to be between 0.1 and $1 \mathrm{~cm}^{-1}$ as we have not tried to optimize this aspect. We defined a cutoff wavelength $\lambda_{c}$ at which the absorption coefficient was equal to $1 \mathrm{~cm}^{-1}$, and propagation losses in excess of $1 \mathrm{~cm}^{-1}$ were therefore expected for wavelengths lower than this one. The cutoff wavelength thus represents the limit over which the structure losses take predominance over the absorption losses of the samples.

In Figure 2 can be seen the absorption coefficient spectra and their linear fit plotted in semilogarithmic 
Table 2. Dye-Matrix Couples ${ }^{a}$

\begin{tabular}{|c|c|c|c|c|c|c|c|}
\hline Dye & Matrix & $\begin{array}{c}\text { Dye } \\
\text { Concentration } \\
\left(10^{-2} \mathrm{~mol} / \mathrm{L}\right)\end{array}$ & $\begin{array}{l}\text { Thickness } \\
(\mu \mathrm{m})\end{array}$ & $\begin{array}{c}\text { Slope }\left(10^{-2}\right. \\
\left.\mathrm{nm}^{-1}\right)\end{array}$ & $\begin{array}{c}\lambda_{c} \\
(\mathrm{~nm}) \\
\end{array}$ & $\begin{array}{c}\lambda_{L} \\
(\mathrm{~nm}) \\
\end{array}$ & $\begin{array}{c}\operatorname{Abs}\left(\lambda_{L}\right) \\
\left(\mathrm{cm}^{-1}\right)\end{array}$ \\
\hline \multirow[t]{4}{*}{$\mathrm{RhB}$} & PMMA15 & 3.3 & 1.9 & 5.4 & 638 & 640 & 0.8 \\
\hline & PMMA120 & 3.2 & 2.6 & 6.0 & 627 & 634 & 0.4 \\
\hline & PMMA350 & 3.2 & 2.1 & 5.2 & 637 & 634 & 1.4 \\
\hline & TCPTEOS & 2.2 & 2.3 & 5.8 & 633 & 634 & 0.9 \\
\hline \multirow{3}{*}{ Rh6G } & PMMA120 & 3.7 & 3.0 & 5.6 & 618 & 606 & 4.5 \\
\hline & PMMA350 & 3.7 & 5.6 & 5.3 & 621 & 612 & 3 \\
\hline & TCPTEOS & 0.24 & 3.4 & 6.3 & 587 & 580 & 2.7 \\
\hline \multirow[t]{3}{*}{ PM597 } & PMMA15 & 3.8 & 2.0 & 5.4 & 602 & 606 & 0.6 \\
\hline & PMMA120 & 4.5 & 2.8 & 5.3 & 602 & 604 & 0.6 \\
\hline & PMMA350 & 4.6 & 6.6 & 5.4 & 601 & 602 & 0.9 \\
\hline \multirow{2}{*}{ DCM } & PMMA350 & 6.4 & 6.4 & 2.8 & 629 & 625 & 1.3 \\
\hline & PVK & 8.4 & 1.9 & 2.2 & 673 & 625 & 10 \\
\hline Pery Red & PMMA120 & 3.2 & 2.5 & 5.8 & 649 & 647 & 1.2 \\
\hline
\end{tabular}

${ }^{a}$ Shown are the dye concentration, thickness, cutoff wavelength $\lambda_{c}$, ASE wavelength $\lambda_{L}$, and extrapolated residual absorption at ASE wavelength $\operatorname{Abs}\left(\lambda_{L}\right)$. The error bars for $\lambda_{c}$ are $\pm 3 \mathrm{~nm}$ for all couples except for the DCM-doped samples, for which the error bars are \pm 6 $\mathrm{nm}$. The error bars for $\lambda_{L}$ are $\pm 1 \mathrm{~nm}$, and the residual absorption is estimated at $\pm 20 \%$.

scale for PM597-doped PMMA120 and PVK matrices. The cutoff wavelengths $\lambda_{c 1}$ and $\lambda_{c 2}$ for PMMA120 and PVK matrices, respectively, are determined. One can see that the slope of the linear fit and the cutoff wavelengths are very different. Indeed, there are more losses in the PVK environment (higher $T_{g}$ ) than in PMMA120 for a given wavelength on the red side of the absorption band, due to induced inhomogeneous broadening. The absorption slope and the cut-

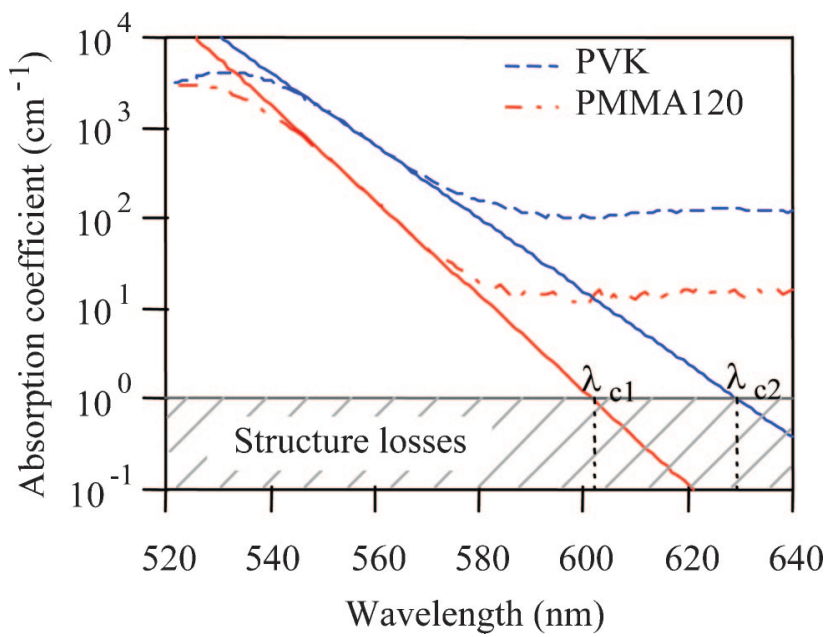

Fig. 2. (Color online) Absorption spectra and fits (solid curves) for PM597-doped PMMA120 and PVK. The respective wavelengths $\lambda_{c 1}$ and $\lambda_{c 2}$, for which the fitted absorption coefficients are equal to the structure losses (estimated at $1 \mathrm{~cm}^{-1}$ ) are the cutoff wavelengths for each dye-matrix couple. off wavelengths thus defined are reported in Table 2 (columns 5 and 6) for all the samples studied in this paper.

\section{B. Amplified Spontaneous Emission}

The thin films were pumped at $532 \mathrm{~nm}$ with the second harmonic of a nanosecond $Q$-switch Nd:YAG laser beam focused with a cylindrical lens (with a waist of approximately $4 \mu \mathrm{m}$ ). To limit the intensity variation in the pump zone, an afocal system of lenses overspreads the incoming beam and a square diaphragm of $25 \mathrm{~mm} \times 25 \mathrm{~mm}$ was used to select the center of the Gaussian beam profile before focusing with the cylindrical lens. The pump zone covered the entire width of the sample $(25 \mathrm{~mm})$. The ASE wavelength could be measured with an OMA (optical multichannel analyser, Acton Research Corporation SpectroPro-275). The ASE wavelengths $\lambda_{L}$ and the extrapolated absorption coefficients at ASE wavelength $\operatorname{Abs}\left(\lambda_{L}\right)$ of the different samples studied are reported in Table 2, in columns 7 and 8.

\section{Propagation Losses}

To measure the propagation losses of the integrated ASE propagating in the dye-doped polymer thin films, the sample is pumped with the same secondharmonic nanosecond $Q$-switch Nd:YAG focused beam as previously in Subsection 3.B, but the pump zone is limited to a $1 \mathrm{~cm}$ width with a slit placed in front of the sample. Thus on each side of the pump zone scattered light can be seen from the propagating ASE beam inside the planar waveguide. The scattered light from a given spot on the sample is propor- 


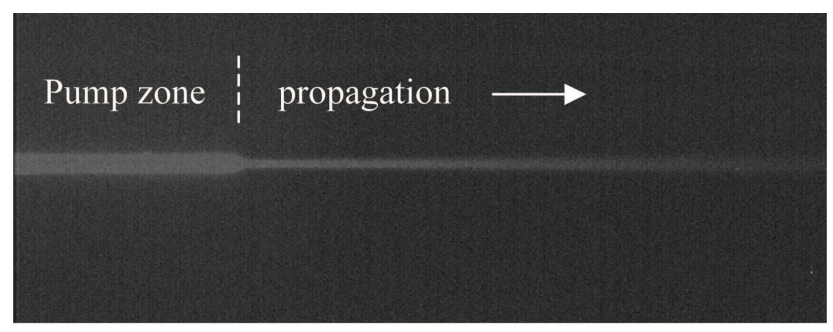

(a)

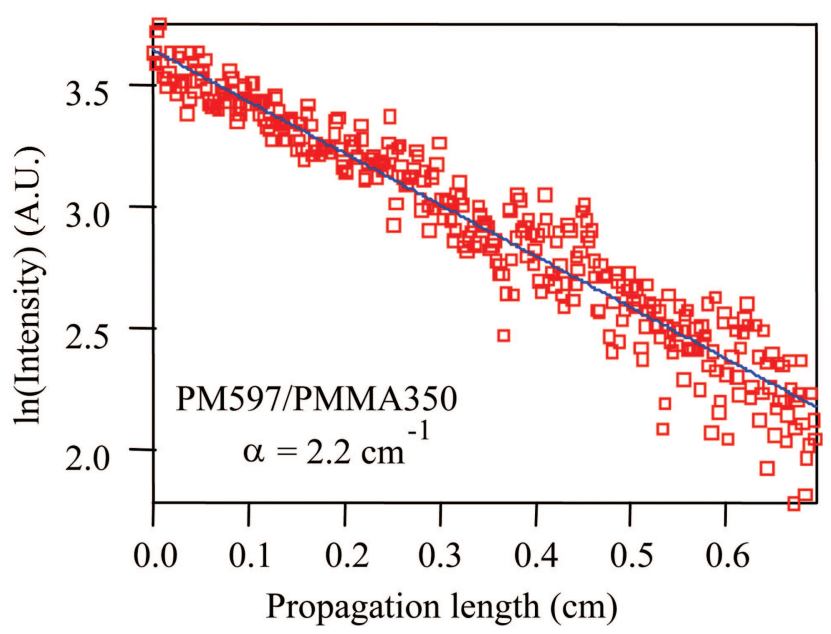

(b)

Fig. 3. (Color online) (a) CCD image of scattered light from propagating amplified spontaneous emission in a thin film, and (b) decrease in propagating intensity (squares) and fit (line) versus propagation length for PM597-doped PMMA350.

tional to the intensity of light propagating in the film. As the light propagates through the thin film, it undergoes losses and so its intensity, and thus the scattered intensity $I$, decreases with propagation length $z$ as follows:

$$
I(z)=I_{0} \exp (-\alpha z)
$$

where $z$ is the distance along the propagation axis in centimeters, $I_{0}$ is the scattered intensity at $z=0$, and $\alpha$ is the propagation loss coefficient in $\mathrm{cm}^{-1}$.

To determine the propagation loss coefficient $\alpha$, an image of the normally scattered light was taken with a CCD camera, as seen in Fig. 3(a), and sent to a Labview program (National Instruments). Regions of interest in the image can be selected, and the decreasing logarithm of the intensity can be plotted against the propagation length as shown in Fig. 3(b). The slope of the linear fit of the data, plotted on a semilogarithm graph, is the measure of the propagation loss coefficient. The measured propagation loss coefficients of the PM597-doped organic polymer samples, ranging from 1.2 to 4.0 for an increasing $T_{g}$ from $100{ }^{\circ} \mathrm{C}$ to $200{ }^{\circ} \mathrm{C}$, are listed in Table 3 . The reproducibility of the results was checked with a second series of samples. We note that the propagation losses were low. Indeed, Lu et al. ${ }^{16}$ reported small propagation
Table 3. Propagation Loss Coefficient for PM597-Doped Organic Polymer Matrices

\begin{tabular}{lcc}
\hline Matrix & $\begin{array}{c}\alpha\left(\lambda_{\mathrm{L}}\right) \\
\left(\mathrm{cm}^{-1}\right)\end{array}$ & $\begin{array}{c}\lambda_{\mathrm{L}} \\
(\mathrm{nm})\end{array}$ \\
\hline PMMA15 & 1.2 & 606 \\
PMMA120 & 1.5 & 604 \\
PMMA350 & 2.2 & 602 \\
PVK & 4.0 & 611 \\
\hline
\end{tabular}

losses of $2.49 \mathrm{~cm}^{-1}$ for a dye-doped polysterene thin film $\left(T_{g}=100^{\circ} \mathrm{C}\right)$.

\section{Discussion}

\section{A. Cutoff and Amplified Spontaneous Emission Wavelengths}

In the case of laser dye-doped polymer thin films, the extrapolated absorption coefficient values that interest us are at emission wavelength, which is redshifted approximately $30 \mathrm{~nm}$ from the absorption peak, well within the $345 \mathrm{~nm}$ maximum extrapolation for which the model used to analyze the absorption spectrum has been validated. ${ }^{15}$ Therefore the model is valid in this case.

The measured ASE wavelength $\lambda_{L}$ has been plotted against the calculated cutoff wavelength $\lambda_{c}$ for all the dye-matrix couples in Fig. 4 . The line represents the case in which $\lambda_{L}=\lambda_{c}$. If the structure losses were well evaluated, and considering that the fluorescence decreases more slowly than the absorption losses with increasing wavelength, the cutoff wavelength is at the point of maximum gain. Indeed, for emission wavelengths higher than $\lambda_{c}$ (above the line), the losses become constant, while the fluorescence still decreases, and for lower emission wavelengths (under the line), the losses increase more steadily than the optical gain (see Fig. 5). We can see in Fig. 4 that for most of the dye-matrix couples studied, the ASE and cutoff wavelengths are equivalent. The wavelength difference $\left(\lambda_{L}-\lambda_{c}\right)$, reported in Table 2, is estimated for all couples with error bars of $\pm 4 \mathrm{~nm}$ except for DCM-doped samples for which the error bars are $\pm 6 \mathrm{~nm}$, as the slope of the absorption coefficient fit is much lower than for the other samples. For the RhB-, PM597-, and Pery Red-doped samples, the wavelength difference is approximately 0 , close to within the error bars, except for PM597/PVK. For the Rh6G-doped and DCM-doped samples, the wavelength difference is negative, which means that the samples emit at wavelengths lower than $\lambda_{c}$. These last samples emit in a wavelength range in which the losses, and in particular the absorption losses, are not negligible. The doped PVK samples (PM597 and DCM) emit at particularly shifted wavelengths. We can see that the ASE wavelength position of the dye-matrix couples depends on the natures of the dye and of the matrix. The DCM and Rh6G dyes and the PVK matrix are not favorable as they lower the value of the slope of the absorption coefficient fit, and that shifts the ASE 


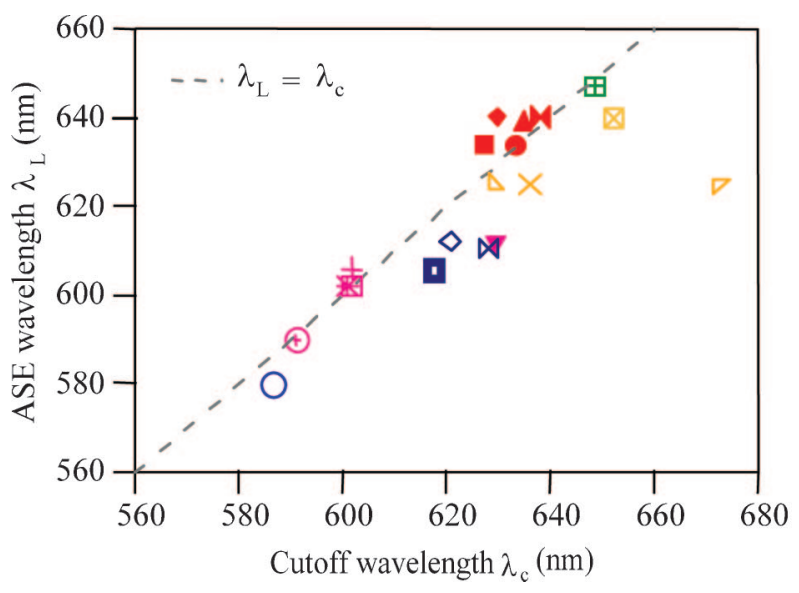

- RhB/PMMA350

$\diamond$ Rh6G/PMMA350

- RhB/PMMA120

- Rh6G/PMMA120

M RhB/PMMA15

$\bowtie$ Rh6G/PMMA15

- RhB/TCPTEOS

Rh6G/TCPTEOS

$\triangle \mathrm{RhB} / \mathrm{PhiTEOS}$

- PM597/PVK

$\mathrm{DCM} / \mathrm{PVK}$

PM597/PMMA350

DCM/PMMA350

PM597/PMMA120

DCM/PMMA120

+ PM597/PMMA15

DCM/PMMA15

Fig. 4. (Color online) ASE versus cutoff wavelength for several dye-host couples.

wavelength into the residual absorption's influence range.

Therefore it appears that for most of the dye-matrix couples, the determination of the cutoff wavelength through the analysis of the absorption spectrum is a powerful tool for the prior estimation of the ASE emission wavelength of dye-doped organic or organomineral polymer thin films.

\section{B. Propagation Losses Versus $T_{g}$}

The propagation loss coefficient has been measured for PM597 doped in four different organic polymer matrices, PMMA15, PMMA120, PMMA350, and PVK, to study the influence of the glass transition temperature $T_{g}$, i.e., the rigidity of the matrix, on the propagation losses of the PM597-doped polymer thin film. The results have been plotted in Fig. 6. One can see that the propagation losses at laser wavelengths increase with the increasing rigidity of the chromophore environment. The extrapolated residual absorption coefficients at laser wavelengths for these thin films have been added to the graph to compare them with the measured propagation losses. One can see that the residual absorption coefficient of doped PMMA is lower than the propagation losses, which is only natural as propagation losses count absorption, scattering, and structure losses among others. This is not true for PVK, but the error bar of $\pm 20 \%$ spreads to

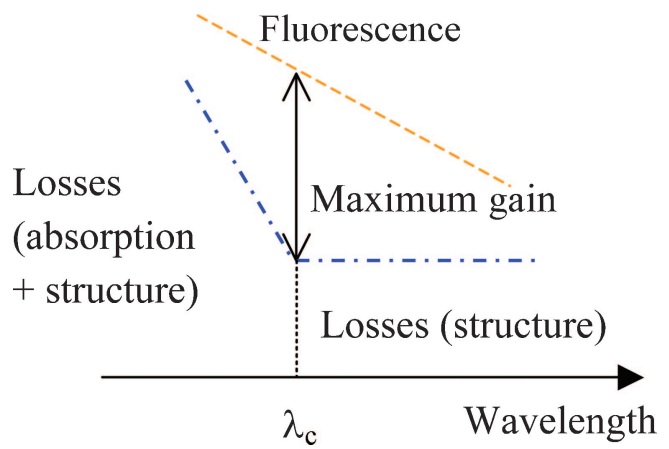

Fig. 5. (Color online) Schematic of maximum gain at cutoff wavelength. lower values. The extrapolated residual absorption coefficient has a tendency to increase with increasing rigidity, but more slowly than the propagation loss coefficient. It is to be noted that for the three matrices of lower $T_{g}$, the extrapolated residual absorption coefficient is lower than the estimated structure losses, which means that their absorption losses are negligible compared to structure losses that were, in fact, slightly underestimated for these samples.

This increase of propagation losses with the increase of matrix rigidity is therefore attributed to inhomogeneous broadening of the absorption spectrum as the dye molecules are blocked in more conformations in the matrix pores. However, we note that no measurement of the film roughness has been made, and the structure of the waveguides has not

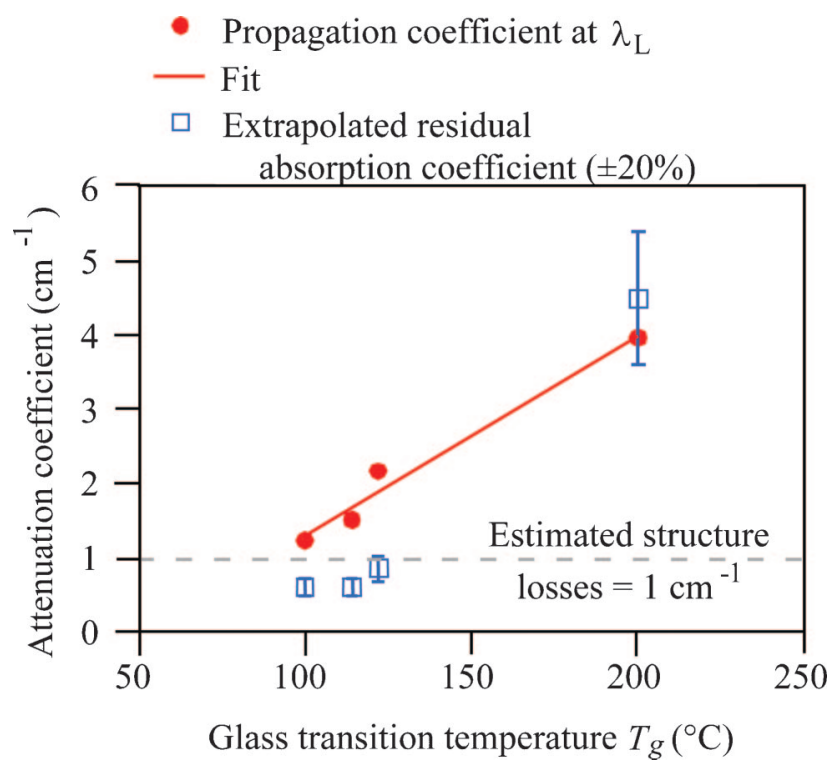

Fig. 6. (Color online) Propagation losses and extrapolated absorption coefficient at ASE wavelength versus glass transition temperature $T_{g}$ of the host environment for PM597 doped in PMMA15 $\left(T_{g}=100{ }^{\circ} \mathrm{C}\right)$, PMMA120 $\left(T_{g}=114^{\circ} \mathrm{C}\right)$, PMMA350 $\left(T_{g}\right.$ $\left.=122^{\circ} \mathrm{C}\right)$, and PVK $\left(T_{g}=200^{\circ} \mathrm{C}\right)$. 
been optimized, so the importance of these factors on propagation losses could not be evaluated.

\section{Conclusion}

We have seen that residual absorption has an important role in the determination of the ASE wavelength of dye-doped, organic, or organomineral, polymer thin films. Through the analysis and extrapolation of the absorption spectrum, a quick and widely available measurement, the ASE wavelength range and the absorption coefficient in the emission range can be estimated. This last point can be of interest in evaluating the absorption losses in the tuning range of an integrated laser device in a distributed feedback configuration for which operational laser wavelengths are imposed. The position of the laser wavelength inside or outside the residual absorption's influence depends on the nature of the dye and the matrix.

Propagation losses at ASE wavelength have also been measured in a series of four PM597-doped organic polymer matrices of different glass transition temperatures. As in the case of the electro-optic chromophores, the absorption propagation losses of such dye-doped material were shown to increase with the increasing rigidity of the host matrix on the red edge of the absorption band.

\section{References}

1. Y. Oki, K. Aso, D. Zuo, N. Vasa, and M. Maeda, "Widewavelength-range operation of a distributed-feedback dye laser," Jpn. J. Appl. Phys. Part 1 41, 6370-6374 (2002).

2. L. Rocha, V. Dumarcher, C. Denis, P. Raimond, C. Fiorini, and J.-M. Nunzi, "Laser emission in periodically modulated thin films," J. Appl. Phys. 89, 3067-3069 (2001).

3. W. Holzer, A. Penzkofer, T. Pertsch, N. Danz, A. Bräuer, E. Kley, H. Tillmann, C. Bader, and H.-H. Hörhold, "Corrugated neat thin-film conjugated polymer distributed-feedback lasers," Appl. Phys. B 74, 333-342 (2002).

4. J. Lawrence, G. Turnbull, and I. Samuel, "Polymer laser fab- ricated by a simple micromolding process," Appl. Phys. Lett. 82, 4023-4025 (2003).

5. D. Wright, E. Brasselet, J. Zyss, G. Langer, and W. Kern, "Dye-doped organic distributed-feedback lasers with index and surface gratings: the role of pump polarization and molecular orientation,” J. Opt. Soc. Am. B 21, 944-950 (2004).

6. M. McGehee and A. Heeger, "Semiconducting (conjugated) polymers as materials for solid-state lasers," Adv. Mater. 12, 1655-1668 (2000).

7. G. Turnbull, P. Andrew, W. Barnes, and I. Samuel, "Operating characteristics of a semiconducting polymer laser pumped by a microchip laser," Appl. Phys. Lett. 82, 313-315 (2003).

8. T. Voss, D. Scheel, and W. Schade, "A microchip-laser-pumped DFB-polymer-dye laser," Appl. Phys. B 73, 105-109 (2001).

9. D. Nilsson, S. Balslev, M. Gregersen, and A. Kristensen, "Microfabricated solid-state dye lasers based on a photodefinable polymer," Appl. Opt. 44, 4965-4971 (2005).

10. X. Peng, L. Liu, J. Wu, Y. Li, Z. Hou, L. Xu, W. Wang, and F. $\mathrm{Li}$, "Wide-range amplified spontaneous emission wavelength tuning in a solid-state dye waveguide," Opt. Lett. 25, 314-316 (2000).

11. D. Lo, L. Shi, J. Wang, G.-X. Zhang, and X.-L. Zhu, "Zirconia and zirconia-organically modified silicate distributed feedback lasers tunable in the visible," Appl. Phys. Lett. 81, 2707-2709 (2002).

12. R. J. Kruhlak and M. G. Kuzyk, "Side-illumination fluorescence spectroscopy. I. Principles,” J. Opt. Soc. Am. B 16, 17491755 (1999).

13. R. J. Kruhlak and M. G. Kuzyk, "Side-illumination fluorescence spectroscopy. II. Applications to squaraine-dye-doped polymer optical fibers," J. Opt. Soc. Am. B 16, 1756-1767 (1999).

14. K. Geetha, M. Rajesh, V. P. N. Nampoori, C. P. G. Vallabhan, and P. Radhakrishnan, "Loss characterization rhidamine 6G doped polymer film waveguide by side illumination fluorescence," J. Opt. A, Pure Appl. Opt. 6, 379-383 (2004).

15. A.-C. Le Duff, V. Ricci, T. Pliska, M. Canva, G. Stegeman, K. Chan, and R. Twieg, "Importance of chromophore environment on the near-infrared absorption of polymeric waveguides," Appl. Opt. 39, 947-953 (2000).

16. W. Lu, B. Zhong, and D. Ma, "Amplified spontaneous emission and gain from optically pumped films of dye-doped polymers," Appl. Opt. 43, 5074-5076 (2004). 